\title{
Plasticity and fracture of aluminium 7075 at high strain rates and elevated temperatures
}

\author{
Xueyang Li, Kedar Pandya, Nikos Karathanasopoulos, Christian C. Roth* and Dirk Mohr \\ Chair of Computational Modelling of Materials in Manufacturing, Department of Mechanical and \\ Process Engineering, Swiss Federal Institute of Technology (ETH), Zurich, Switzerland
}

\begin{abstract}
Slow, intermediate and high strain rate experiments with UT geometries are performed on aluminum AA7075-T6 sheet metal at various temperatures. The comprehensive experimental program characterizes the plasticity response at temperatures ranging from $20^{\circ} \mathrm{C}$ to $360^{\circ} \mathrm{C}$ and at strain rates ranging from $0.001 / \mathrm{s}$ to $150 / \mathrm{s}$. The elevated temperature - elevated strain rate experiments are performed on a hydraulic tensile testing machine and a Split Hopkinson Pressure Bar system with a Load Inversion Device along with a custom-made induction heating system. A machine learning based modified Johnson-Cook plasticity model is calibrated to capture the complex strain rate and temperature effect of the observed hardening response.
\end{abstract}

\section{Introduction}

There is a constant quest to better understand and model the effect of strain rate and temperature driven by industrial problems including metal forming, machining, accidental loading or high-speed impact, amongst others. Aluminium alloy AA 7xxx series has seen particular interest for impact protection systems [1]. These alloys often feature minimum strain rate effect at room temperature [2] but high strain rate dependency at elevated temperatures [3]. To characterize these complex effects, many phenomenological models have been developed, most notably the Johnson-Cook (JC) model [4]. These mechanisminspired models have seen wide applications over the years. More recently data driven models based on various machine-learning algorithms have been shown to accurately describe the mechanical response of materials $[5,6]$.

In this study, uniaxial tensile experiments are performed covering strain rates from $0.001 / \mathrm{s}$ to $110 / \mathrm{s}$ and temperatures from $20^{\circ} \mathrm{C}$ to $360^{\circ} \mathrm{C}$. A neural network (NN) based hardening model is identified to describe the strain rate and temperature dependence. The performance of the NN model is compared to that of a classic JC model to highlight the flexibility and accuracy of the NN modelling.

\footnotetext{
* Corresponding author: ccroth@ethz.ch
} 


\section{Material and specimens}

The material investigated in this study is a $2 \mathrm{~mm}$ thick high-strength aluminium alloy AA7075-T6. All specimens are extracted through waterjet cutting and Fig. 1 shows the two uniaxial tensile (UT) geometries used.

- Uniaxial tension specimens featuring a $10 \mathrm{~mm}$ wide and 40mm long gauge section (Fig. 1a) for low and intermediate strain rates.

- Short uniaxial tension specimens (D-UT) with $15 \mathrm{~mm}$ long and $5 \mathrm{~mm}$ wide gauge section used for testing at high strain rates (Fig. 1b).

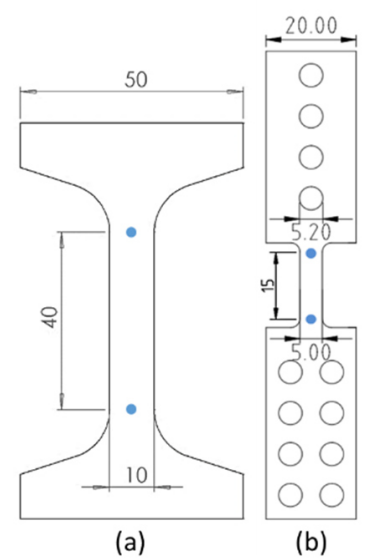

Fig. 1. Specimen geometries for (a) low, intermediate, and (b) high strain rate testing. The blue dots mark positions of extensometers for global displacement measurements through DIC.

\section{Experimental Methods}

\subsection{Setup for low strain rates}

Experiments at low strain rates are conducted on a hydraulic universal testing machine (Instron 8801) using displacement control. An actuator speed of $2.4 \mathrm{~mm} / \mathrm{min}$ is selected, corresponding to an engineering strain rate of $0.001 / \mathrm{s}$. The specimens are spray painted with a random white and black speckle pattern for digital image correlation (VIC2D, Correlated Solution). The images are acquired at $1 \mathrm{~Hz}$ using a 5MP camera (Point Grey GS3- U351S5M-C). A custom made induction heating system with pyrometer based temperature control is employed. The specimens are heated up to target temperature at rate of $30 \mathrm{~K} / \mathrm{s}$. An infrared camera (FLIR x6801) is used to verify the homogeneity of the temperature field.

\subsection{Setup for intermediate strain rates}

Experiments at intermediate strain rates are carried out on the same hydraulic universal testing machine (Instron 8801). A loading speed of $240 \mathrm{~mm} / \mathrm{min}$ is chosen, which is 100 times the speed of the slow tests. Images for DIC are acquired using a high speed camera (Photron SA-Z) equipped with a $105 \mathrm{~mm} \mathrm{f} 2.8$ macro lens at a frequency of $200 \mathrm{~Hz}$. The image and data acquisition is triggered by the rise in the force signal. The same induction heating system and high speed IR camera are used for temperature control. 


\subsection{Setup for high strain rates}

Experiments at high loading speeds are conducted on a Split Hopkinson Pressure Bar (SHPB) system with a Load Inversion Device (LID) for tensile testing. The details on the SHPB system is described in [7]. The specimens are mounted with $12 \times$ M5 screws. The axial force acting on the specimen is calculated from the strain gauge signals obtained from the output bar at a distance of $430 \mathrm{~mm}$ from the specimen/bar interface.

The displacement is measured using the same high-speed camera as for the intermediate strain rate experiments (Photron SA-Z). The camera is set to a reduced resolution of $512 \times 280$ pixels operating at $100 \mathrm{kHz}$. The image acquisition and data acquisition is triggered simultaneously by the rise in the input bar signal.

\section{Constitutive modelling}

An elasto-plastic framework is chosen to model the AA7075-T6. The Young's modulus and Poisson ratio are listed in Table 1. In the absence of information on the material anisotropy, a von-Mises yield surface with associated flow rule is selected for simplicity. The yield function writes:

$$
f[\sigma, k]=\bar{\sigma}_{r M}-k=0
$$

The rate and temperature dependent plastic response is described with two distinct models: an analytical Johnson-Cook type of model and a neural network based hardening model.

\subsection{Johnson-Cook type hardening law}

The Johnson-Cook type formulation multiplicatively decomposes the deformation resistance $k$ into a strain, a strain rate and a temperature term:

$$
k\left[\bar{\varepsilon}_{p}, \dot{\bar{\varepsilon}}_{p}, T\right]=k_{S V}\left[\bar{\varepsilon}_{p}\right] k_{\bar{\varepsilon}_{p}}\left[\dot{\bar{\varepsilon}}_{p}\right] k_{T}[T]
$$

Here the strain hardening term is a linear combination of Swift and Voce term with weighting parameter $\alpha$.

$$
\begin{aligned}
& k_{S V}\left[\bar{\varepsilon}_{p}\right]=\alpha k_{S}\left[\bar{\varepsilon}_{p}\right]+(1-\alpha) k_{V}\left[\bar{\varepsilon}_{p}\right] \\
& k_{S}\left[\bar{\varepsilon}_{p}\right]=A\left(\varepsilon_{0}+\bar{\varepsilon}_{p}\right)^{n} \\
& k_{V}\left[\bar{\varepsilon}_{p}\right]=k_{0}+Q\left(1-e^{-\beta \overline{\varepsilon_{v}}}\right)
\end{aligned}
$$

The strain rate and temperature terms are taken as the original JC type, respectively.

$$
\begin{aligned}
& k_{\dot{\bar{\varepsilon}}_{\bar{p}}}\left[\dot{\bar{\varepsilon}}_{p}\right]=\left\{\begin{array}{lll}
1 & \text { for } & \dot{\bar{\varepsilon}}_{p}<\dot{\varepsilon}_{0} \\
1+C \ln \left[\frac{\dot{\dot{\varepsilon}_{p}}}{\dot{\bar{\varepsilon}}_{0}}\right] & \text { for } & \dot{\bar{\varepsilon}}_{p} \geq \dot{\varepsilon}_{0}
\end{array}\right. \\
& k_{T}[T]=\left\{\begin{array}{lll}
1 & \text { for } & T<T \\
1-\left(\frac{T-T_{r}}{T_{m}-T_{r}}\right)^{m} & \text { for } & T \leq T \leq T_{r}
\end{array}\right.
\end{aligned}
$$




\subsection{Neural network based hardening law}

The neural network based hardening model follows closely the formulation of [6]. The deformation resistance is decomposed into a reference mixed Swift-Voce strain hardening, as well as a neural network term describing the effect of strain rate and temperature.

$$
k\left[\bar{\varepsilon}_{p}, \dot{\bar{\varepsilon}}_{p}, T\right]=k_{S V}\left[\bar{\varepsilon}_{p}\right] k_{N N}\left[\bar{\varepsilon}_{p}, \dot{\bar{\varepsilon}}_{p}, T\right]
$$

The neural network in this study is a feed-forward fully connected NN with three hidden layers, each with 10 neurons. The output of the $k_{N N}$ term is a scaling factor that scales the reference hardening curve to the current rate and temperature.

\section{Results and discussions}

\subsection{Experimental Results}

Figure 2a shows the experimentally-measured engineering stress-strain curves from the uniaxial tensile specimens at various loading speeds and temperatures. The corresponding true stress-plastic strain curves are shown in Fig. $2 b$ up to the point of uniform elongation.

A monotonic and negative effect of the temperature on the equivalent stress is observed irrespective of the strain rate. The effect of strain rate is negligible below a temperature of $60^{\circ} \mathrm{C}$. Only starting from $120^{\circ} \mathrm{C}$ a significant strain rate hardening response is observed. At $360^{\circ} \mathrm{C}$, a rise in yield stress of more than $90 \%$ is obtained when increasing the loading speed from $0.001 / \mathrm{s}$ to $0.1 / \mathrm{s}$.

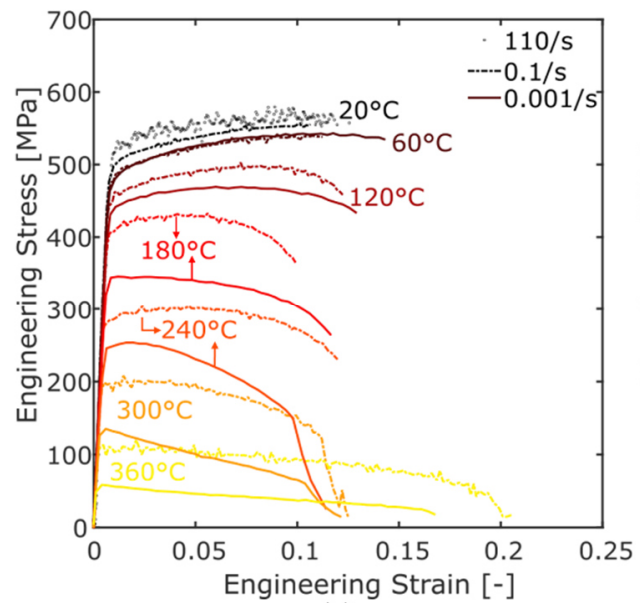

(a)

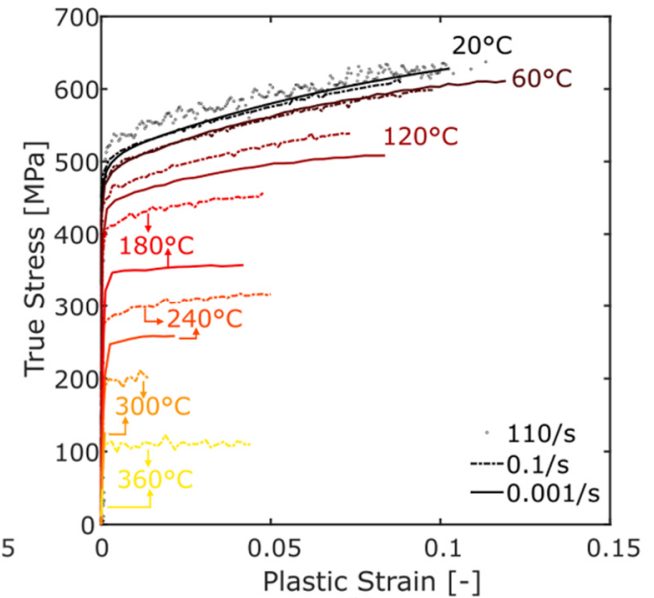

(b)

Fig. 2. (a) Engineering stress-strain curves from uniaxial tensile tests at various temperatures. The low speed tests are highlighted as solid lines. The intermediate speed tests are plotted in dashed lines and the high strain rate tests are marked in dots. (b) True stress-plastic strain curves from uniaxial tensile tests at various temperatures. The curves are truncated at the point of force maximum (uniform elongation).

\subsection{Modeling results}

The Young's modulus and Poisson's ratio are obtained from the engineering stress-strain curve of the room temperature UT test. The parameters for the reference mixed Swift-Voce 
strain hardening are identified by fitting the UT true stress plastic strain curve at room temperature. Table 1 provides an overview of the base model parameters.

The JC parameters are identified on the true stress-plastic strain curves for the different strain rates and temperatures. The details of the calibrated model parameters are listed in Table 2.

The NN based hardening model is calibrated using the same true stress-plastic strain curves as for the $\mathrm{JC}$ calibration in conjunction with a resilient back-propagation (RPROP) [8] approach. The model is trained for overall 500 epochs, taking $<10$ min on a desktop PC.

Table 1. Plasticity model parameters for AA7075-T6

\begin{tabular}{lllllll}
\hline \hline $\begin{array}{c}E \\
{[\mathrm{GPa}]}\end{array}$ & $v$ & $\rho$ & & & & \\
\hline 67.6 & 0.33 & 2780 & & & & \\
\hline \hline$A$ & $\varepsilon_{0}$ & $n$ & $k_{0}$ & $Q$ & $\beta$ & $\alpha$ \\
{$[\mathrm{MPa}]$} & {$[-]$} & {$[-]$} & {$[\mathrm{MPa}]$} & {$[\mathrm{MPa}]$} & {$[-]$} & {$[-]$} \\
\hline 855 & 0.026 & 0.15 & 502 & 194 & 10.08 & 0.5 \\
\hline \hline
\end{tabular}

Table 2. Johnson-Cook type hardening parameters

\begin{tabular}{ccccc}
\hline \hline$C$ & $\dot{\varepsilon}_{0}$ & $m$ & $T_{r}$ & $T_{m}$ \\
{$[-]$} & {$[1 / \mathrm{s}]$} & {$[-]$} & {$\left[{ }^{\circ} \mathrm{C}\right]$} & {$\left[{ }^{\circ} \mathrm{C}\right]$} \\
\hline 0.011 & 0.001 & 1.23 & 20 & 477 \\
\hline \hline
\end{tabular}

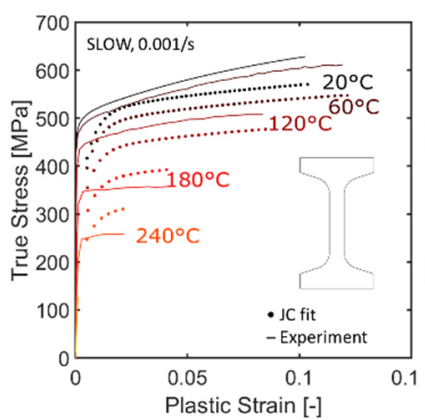

(a)

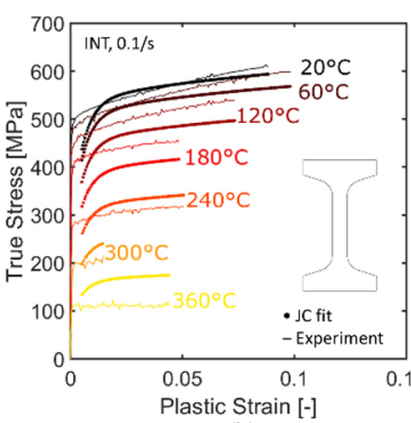

(b)

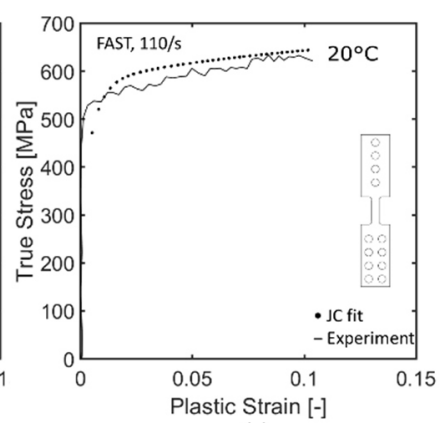

(c)

Fig. 3. Johnson-Cook model predicted hardening function (dots) against the experimental curves (solid lines).

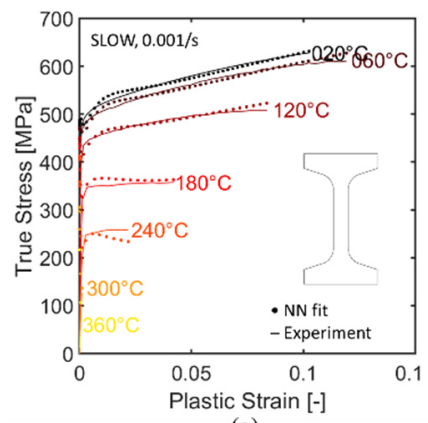

(a)

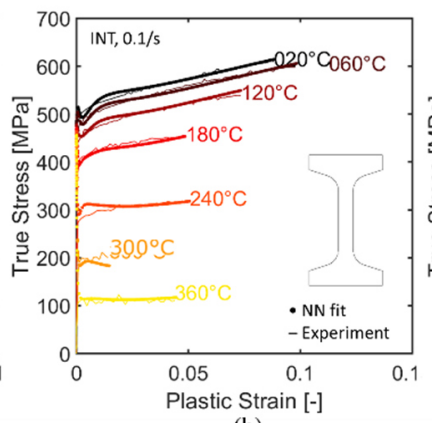

(b)

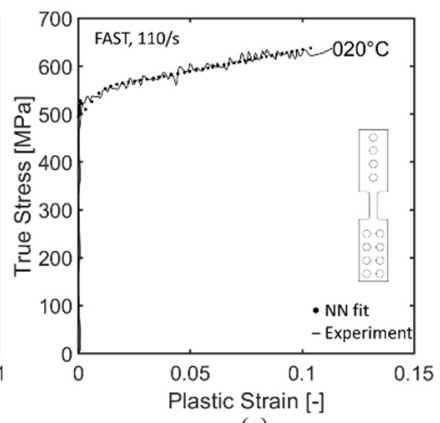

(c)

Fig. 4. Neural Network based hardening function (dots) plotted against the experimental curves (solid lines). Note the model's high flexibility in dealing with curves with significant strain hardening at lower temperatures and moderate strain hardening at higher temperatures. 
Comparing the calibrated JC model (Fig. 3) to the NN model (Fig. 4), the NN model outperforms the JC formulation in such regression tasks by a significant amount. The JC model is limited by its decomposition of the strain, strain rate and temperature term, which enforces a constant percentage of strain rate effect for all temperatures. Consequently, it is not possible to capture the negligible strain rate effect at low temperatures and the significant strain rate effect at elevated temperatures above $120^{\circ} \mathrm{C}$. In this case the $\mathrm{NN}$ model serves as a universal function approximator, unveiling the intrinsic connections between the input and output terms.

\section{Conclusion}

An experimental investigation on the aluminium alloy AA7075-T6 is carried out to characterize its response at various strain rates and temperatures. A machine learning based plasticity model is employed which decomposes the deformation resistance into a reference Swift-Voce strain hardening, and a neural network based rate and temperature dependency. It is demonstrated that the NN model achieves a significantly higher prediction accuracy than a classic JC type formulation.

\section{References}

1. T. Børvik, H. Aunehaugen, O. S. Hopperstad, "Impact behaviour of the high-strength aluminium alloy AA7075-T651" DYMAT - International Conference on the Mechanical and Physical Behaviour of Materials under Dynamic Loading, 695-701 (2009)

2. C. C. Roth, T. Fras, and D. Mohr, "Dynamic perforation of lightweight armor: Temperature-dependent plasticity and fracture of aluminum 7020-T6," Mech. Mater., vol. 149, no. July, p. 103537, 2020

3. K. S. Pandya, C. C. Roth, and D. Mohr, "Strain rate and temperature dependent fracture of aluminum alloy 7075: Experiments and neural network modeling," Int. J. Plast., vol. 135, p. 102788, (2020)

4. G. Johnson and W. Cook, Engineering Fracture Mechanics, vol. 21, no. 1, pp. 31-48 (1985)

5. U. Ali, W. Muhammad, A. Brahme, O. Skiba, and K. Inal, "Application of artificial neural networks in micromechanics for polycrystalline metals,” Int. J. Plast., vol. 120, no. November 2018, pp. 205-219, 2019

6. X. Li, C. C. Roth, and D. Mohr, "Machine-learning based temperature- and ratedependent plasticity model: Application to analysis of fracture experiments on DP steel,” Int. J. Plast., vol. 118, no. October 2018, pp. 320-344, 2019

7. C. Roth and D. Mohr, International Journal of Plasticity, vol. 56, pp. 19-44 (2014)

8. M. Riedmiller and H. Braun, IEEE International Conference on Neural Networks, vol. 1, pp. 586--591 (1993) 